\title{
The selective processing of emotional visual stimuli while detecting auditory targets: An ERP analysis
}

\author{
Harald T. Schupp ${ }^{a, *}$, Jessica Stockburger ${ }^{a}$, Florian Bublatzky $^{a}$, Markus Junghöfer $^{b}$, \\ Almut I. Weike ${ }^{\mathrm{c}}$, Alfons O. Hamm ${ }^{\mathrm{c}}$ \\ ${ }^{a}$ Department of Psychology, University of Konstanz, Germany \\ ${ }^{\mathrm{b}}$ Institute for Biomagnetism and Biosignalanalysis, Münster University Hospital, Germany \\ ${ }^{\mathrm{c}}$ Department of Psychology, University of Greifswald, Germany
}

\section{A R T I C L E I N F O}

Article history:

Accepted 2 July 2008

Available online 15 July 2008

\section{Keywords:}

Emotion

Attention

Event related potential

Competition

EPN

P3

\begin{abstract}
A B S T R A C T
Event related potential studies revealed an early posterior negativity (EPN) for emotional compared to neutral pictures. Exploring the emotion attention relationship, a previous study observed that a primary visual discrimination task interfered with the emotional modulation of the EPN component. To specify the locus of interference, the present study assessed the fate of selective visual emotion processing while attention is directed towards the auditory modality. While simply viewing a rapid and continuous stream of pleasant, neutral, and unpleasant pictures in one experimental condition, processing demands of a concurrent auditory target discrimination task were systematically varied in three further experimental conditions. Participants successfully performed the auditory task as revealed by behavioral performance and selected event related potential components. Replicating previous results, emotional pictures were associated with a larger posterior negativity compared to neutral pictures. Of main interest, increasing demands of the auditory task did not modulate the selective processing of emotional visual stimuli. With regard to the locus of interference, selective emotion processing as indexed by the EPN does not seem to reflect shared processing resources of visual and auditory modality.
\end{abstract}

\section{Introduction}

Preparation for action has been suggested as a key function of emotion (Lang et al., 1997; Öhman et al., 2000). However, because of the flexible tailoring of responses to environmental demands in humans, the organization of successful approach and avoidance behaviors is fostered by the accurate perceptual analysis of the environment (Lang et al., 1997; Öhman et al., 2000). Accordingly, stimuli signifying evolutionarily relevant topics such as food, mating partners, or signals of threat are effective cues to capture selective visual attention. A large array of behavioral (Mathews and MacLeod, 1994; Mogg and Bradley, 1999; Öhman et al., 2000) and neuroimaging studies (e.g., Junghöfer et al., 2005; Lang et al., 1998; Sabatinelli et al., 2004) support the hypothesis that emotional stimuli capture attentional resources. Event related brain potential studies have been used to detail the temporal dynamics of selective emotion processing in visual associative brain cortices.

A number of studies have consistently observed an early ERP difference in processing emotional (pleasant and unplea sant), compared to neutral, pictures. Specifically, processing of emotional stimuli is associated with an early posterior nega

\footnotetext{
* Corresponding author. Institute of Psychology, University of Konstanz, PO Box D36, 78457 Konstanz, Germany. Fax: +49 7531882971.

E mail address: Harald.Schupp@uni konstanz.de (H.T. Schupp).
} 
tivity (EPN) which is most pronounced in a time window from 200300 ms poststimulus (Schupp et al., 2006). Estimating the generator sources of the differential ERP activity support the notion that emotional stimuli receive enhanced processing in posterior visual brain regions (Junghöfer et al., 2001; Schupp et al., 2003). Recent studies extended these findings to other emotional stimulus materials such as emotional faces, words, and hand gestures (Schupp et al., 2004a; Kissler et al., 2007; Flaisch, Schupp, Renner, and Junghöfer, submitted). Accord ingly, the EPN may serve as an early brain potential measure of the prioritized perceptual processing of emotional stimuli. A reliable processing advantage of positive and negative rein forces during initial perception will presumably carry over to later processes assuring that emotional stimuli attract sus tained attention and appropriate actions are undertaken. From this perspective, the preferential processing of emo tional cues seems highly adaptive, ultimately promoting survival and reproductive success (Lang et al., 1997; Öhman et al., 2000).

In a world where various stimuli compete for attentional resources, it is of interest to determine the extent to which the prioritized perceptual processing of emotional stimuli reflects an automatic phenomenon. Previous studies showed that the emotional EPN modulation evinces several characteristics of an automatic process. Specifically, the emotional EPN mod ulation occurred spontaneously, independent from stimulus novelty, and while participants held unrelated task goals in mind (Schupp et al., 2003; Schupp et al., 2006). Noteworthy, the notion of automaticity was not supported when exploring the preferential emotion processing in the context of competing attention demands. A recent study asked participants to count target stimuli defined by line orientation (vertical or horizon tal), which were overlaid on (task irrelevant) pleasant, neutral, and unpleasant IAPS pictures (Schupp et al., 2007a). In demanding task conditions, pleasant and unpleasant pictures failed to elicit increased EPN amplitudes compared to neutral images. Furthermore, interference effects in visual processing can also extend in time as demonstrated by recent research observing attenuated EPN amplitudes of current pictures when preceded by emotional compared to neutral picture contents (Flaisch et al., 2008). Overall, while the EPN modula tion occurs unintentional, autonomous, and involuntary, the emotional EPN effect is modulated by visual attentional demands. Thus, the emotional EPN modulation represents a weakly rather than strong automatic process (Kahneman and Treisman, 1984).

Conceptually similar interference effects emerged when the primary task manipulated space based rather than feature based attention (Vuilleumier et al., 2001; Anderson et al., 2003; Pessoa 2002b). In particular, ERP measures revealed that emotional interference effects may occur at early processing stages. For instance, differential processing of fearful compared to neutral faces in attended conditions were revealed already by the N1 component, but were absent when attention was diverted (Holmes et al., 2003). Similarly, early ERP differences in processing peripherally presented emotional and neutral pictures varied as a function of demand by a centrally presented primary task (Doallo et al., 2006). Overall, these findings suggest that the prioritized perceptual processing of emotional cues is modu lated by attentional manipulations regarding objects and locations.

While emotional interference effects have been consis tently revealed, the locus of interference remains to be determined. Previous research observed interference effects on visual emotion processing in the context of a competing visual task. Thus, these studies do not allow to decide whether interference effects are primarily due to intramodal competi tion for limited perceptual resources or are a more general phenomenon, reflecting the supramodal sharing of processing resources. To specify the locus of interference, the present study investigated the processing of emotional pictures in the context of a primary auditory attention task.

Previous research exploring the processing of irrelevant crossmodal stimuli is consistent with the hypothesis of a supramodal locus of interference. For instance, modulations in the processing of secondary to be ignored probe stimuli were assessed while participants viewed pictures varying in hedo nic valence. Consistently, the P3 to acoustic startle probes was reduced when participants viewed emotional compared to neutral pictures (Cuthbert et al., 1998; Schupp et al., 2004b). Recently, a similar effect was observed when acoustic startle probes were presented in the context of emotional sounds (Keil et al., 2007). It is thought that emotional compared to neutral pictures draw more attentional resources, leaving few resources available for secondary probe processing. Most importantly, observing similar P3 effects for probes presented in the same or different modality as the primary stimuli suggests a supramodal locus of interference. Focusing on earlier ERP components, a further study showed that the mismatch negativity elicited by auditory deviants embedded in a train of standard tones was attenuated when viewing pleasant compared to neutral and unpleasant pictures (Sur akka et al., 1998). Conceptually similar findings emerged in research of crossmodal stimulus processing utilizing explicit attention tasks. Specifically, manipulations of attentional load modulated early and late ERP components to crossmodal task irrelevant stimuli (Woldorff et al., 1991; Hackley, 1993; Kramer et al., 1995). Based on these results, a demanding auditory attention task may interfere with the attention capture of (task irrelevant) emotional visual stimuli as indexed by the EPN.

The notion of distinct pools of resources for the perceptual modalities provides a different perspective (Wickens, 1980). Specifically, interference effects are suspected to primarily depend on processing demands within the same perceptual modality. Recent studies of emotion interference are consis tent with this notion in that emotional and attentional stimuli were presented in the visual modality, presumably competing for limited visual processing resources. Specifically, the higher the attentional load of the primary task, the less resources are available for emotional stimulus processing causing the inter ference in selective emotion processing (Pessoa et al., 2002a; Schupp et al., 2007b). Corroborating evidence is provided by cognitive studies showing that distractor stimuli were pro cessed more efficiently (i.e., in terms of interfering with the primary task and brain activity in perceptual areas) in low compared to high attentional load conditions (reviewed in Lavie, 2005). Conversely, when emotional and attentional stimuli are presented in different modalities, as investigated 
here, one may assume that each stimulus has access to a dis tinct pool of resources. Thus, selective visual emotion proces sing should be unaffected by concurrent auditory task demands.

Cognitive research already provides empirical evidence for the notion that primary tasks did not modulate cross modal stimulus processing. For instance, a recent ERP study observed that the difficulty of primary visual tracking task did not affect the mismatch negativity and P3a component elicited by auditory deviants (Muller Gass et al., 2007). Fur thermore, a fMRI study revealed that auditory task difficulty did not modulate perceptual processing of task irrelevant visual motion stimuli in area MT implicated in motion pro cessing (Rees et al., 2001). However, other studies assessing these responses in similar paradigms reported interference effects of crossmodal task irrelevant stimuli by primary processing demands (Berman and Colby, 2002; Johnson and Zatorre, 2005). For instance, Johnson and Zatorre (2005) observed that attention to the visual or auditory modality is accompanied by a decreased activity in task irrelevant sensory cortices. Methodological differences have been sug gested to account for conflicting results. Comparing across different levels of task demand rather than against passive baseline condition has been sketched as one important variable in explaining divergent findings, with the former providing a more stringent control for task demands (Lavie, 2005). Furthermore, the design should be optimized to prevent the switching of attention across modalities (Mul ler Grass et al., 2007). These concerns were considered in the present study by varying task demand and preventing atten tion switching by concurrent presentation of visual and auditory stimuli.

The present study aimed to investigate whether the early differential processing of emotional stimuli is affected by competing task demands in the auditory domain, whether such effects appear similar for pleasant and unpleasant stimulus materials, and whether the pattern of findings is observed for highly arousing emotional stimuli. To study crossmodal task demands on emotion processing, a visual target discrimination task, which provoked pronounced affective interference effects in a previous study (Schupp, et al., 2007b), was transferred to the auditory domain. While participants viewed a rapid $(3 \mathrm{~Hz})$ and continuous stream of pleasant, unpleasant, and neutral pictures, a concurrent auditory target discrimination task was construed by pre senting two different tone stimuli time locked to picture onset (see Fig. 1). In each of the task conditions, the parti cipants' task was to silently count tone stimuli of either high or low frequency (balanced across participants). Varying the proportion of task relevant tones presented in the stream of IAPS pictures across three experimental conditions (10\%, 50\% or $100 \%$ probability, respectively), task demand was system atically varied. In an additional condition ( $0 \%$ task), IAPS pictures were presented without any task relevant stimuli to replicate previous studies in which the pictures were passively viewed. The order of the four experimental condi tions was balanced across participants. A first set of analyses focused on the target discrimination task using behavioral performance and selected ERP components as dependent measures to assure that participants conducted the task appropriately. A second set of analyses determined whether

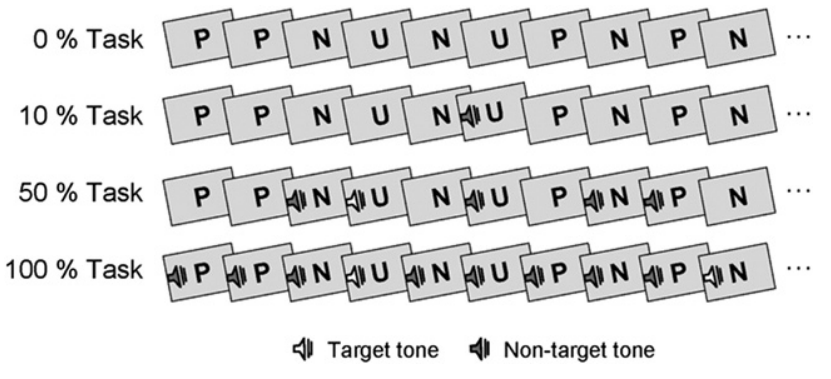

Fig. 1 - Schematic illustration of the four experimental conditions. In one condition, participants passively viewed the pictures $(0 \%$ task). Three further conditions included an auditory target discrimination task with task-related tones presented at $10 \%, 50 \%$ or $100 \%$ of the trials. Twenty percent of all tones in each task condition were target tones and both, frequent and target tones were presented equally often during pleasant $(\mathrm{P})$, unpleasant $(\mathrm{U})$ and neutral (N) picture presentations. In all conditions, pictures were presented as rapid and continuous stream.

selective emotion processing as indexed by the EPN is subject to interference by a concurrent auditory attention task.

\section{2. $\quad$ Results}

\subsection{Explicit attention task: Behavioral and ERP results}

Performance in the primary feature based counting task was captured by an overall error score calculated separately for each of the three task conditions. Overall, behavioral perfor mance was good with an average of $1.8 \%$ errors across the three task conditions. Furthermore, repeated measure ANOVA indicated that performance was comparable across task conditions $\left(M_{10 \%}\right.$ 1.4, SD $4.0 ; M_{50 \%} \quad 2.0, S D \quad 1.8 ; M_{100 \%} \quad 1.9$, SD 2.7), Task $F(2,14) \quad 0.2$, ns.

Participants' ratings of the difficulty to perform the experi mental task indicated that task demand varied across experi mental conditions, Task $F(3,13)$ 28.2, $p<.0001$. A monotonic increase in perceived difficulty was observed from $0 \%, 10 \%, 50 \%$, and $100 \%$ task conditions, $\left(M_{0 \%}\right.$ 13.8, SD 18.6; $M_{10 \%} 45.0$, SD 22.5; $M_{50 \%}$ 54.4, SD 22.8; $M_{100 \%} \quad 66.9$, SD 21.5), Task $F_{\text {lin }}$ $(1,15) 92.4, p<.0001)$. Post hoc tests indicated that each task block was perceived as more difficult than the $0 \%$ task condition, ts $(15)>4.9, p s<.001$. The $100 \%$ condition was perceived as more difficult than the other three conditions, ts $(15)>2.4$, ps $<.05$. Furthermore, while the $50 \%$ task condition was on average perceived as more difficult than the $10 \%$ task condition, this comparison did not reach significance level, t(15) $\quad 1.9, p$.08.

Previous ERP studies of selective attention tasks estab lished that the processing of target stimuli is associated with a pronounced P3 wave (Johnson, 1988). Consistent with these findings, the P3 component was enlarged to target compared to non target stimuli in both, the $50 \%$ (M 3.0 vs. $0.6 \mu \mathrm{V})$ and $100 \%(\mathrm{M} 1.7$ vs. $0.7 \mu \mathrm{V})$ task conditions, Task Fs $(1,15) 34.0$ and 16.6, ps $<.001$, respectively. No significant effects involving Laterality were observed. 


\subsection{The interaction of emotion and attention}

The present data replicated previous findings regarding the modulation of EPN as a function of emotional content, Valence $F(2,14) \quad 40.4, p<.0001$. Pleasant and unpleasant pictures were associated with increased EPN amplitudes compared to neu tral contents, ts(15) 7.8 and 2.6, ps $<.05$, respectively. In addition, as in previous studies (Schupp et al., 2007a), pleasant pictures elicited greater EPN amplitudes than unpleasant contents, $\mathrm{t}(15) \quad 3.2, p<.01$.

Of main interest for the present study, the interaction of Valence (pleasant, neutral, and unpleasant) and Task $(0,10,50$, and $100 \%$ condition) did not reach significance, $F(6,10) 3.0$, $p$.06. In addition, the main effect Task was also not signi ficant, $F(3,13)$ 1.2, ns. Fig. 2 allows to evaluate whether emo tion processing varies as a function of task demand. Specifically, Fig. 2A displays ERP waveforms of a right hemi spheric occipital sensor to inspect experimental conditions for the occurrence of enlarged EPN for the processing of emotio nal stimuli, developing around $150 \mathrm{~ms}$ and most pronounced between 200 and 300 ms after stimulus onset. Complementary evidence regarding the spatial topography of the EPN effect is provided in Fig. 2B, which is typically observed as bilateral and broadly distributed posterior negativity when calculating the difference between emotional and neutral pictures. Most importantly, emotional differentiation did not vary system atically as a function of task demand and was similar for low (10\%) and high demanding (50 and 100\%) task conditions.
For exploratory reasons, emotional modulation was deter mined for each experimental condition in separate ANOVAs. Analyses of all four conditions revealed highly significant main effects of Valence, $F s(2,14) \quad 29.7,11.5,9.9$ and 8.8, $p s<.005$ for the $0 \%, 10 \%, 50 \%$, and $100 \%$ task condition, respectively. Pleasant pictures elicited enlarged EPN amplitudes compared to neutral contents in all conditions, ts $(1,15)>4.2, p s<.001$. Unpleasant images were associated with larger EPN ampli tudes than neutral materials in the $0 \%, 50 \%$, and $100 \%$ task condition, ts $(1,15) \quad 2.7,2.1$, and 2.3, ps $\leq .05$, while being not significant in the $10 \%$ task condition. Furthermore, larger EPN amplitudes to pleasant than unpleasant images were observed in the $0 \%$ and $10 \%$ conditions, ts $(1,15) \quad 3.3$ and $3.3, p s<.01$.

Analyzing pleasant, neutral, and unpleasant pictures separately as a function of task conditions provides a further way to explore the interaction of emotion and attention. For pleasant materials, the interaction of Task and Laterality was significant, $F(3,13) \quad 5.2, p<.01$. Follow up tests revealed task condition effects for the right posterior EPN sensor cluster, $F(3,13) 11.3, p<.001$, while no task effects were observed for the left side, $F(3,13) \quad 0.8$, ns. Post hoc tests conducted for right sensor cluster revealed that passive viewing was associated with larger EPNs compared to the $10 \%$ and $50 \%$ condition, ts $(1,15) \quad 2.1$ and $5.2, p \leq .05$, while no significant differences were observed among the three task conditions, ts $(1,15)<1.6$, ns. For unpleasant and neutral materials, neither the main effect Task nor the interaction Task $\times$ Laterality reached significance, $F s(3,13)<1.6$, ns.
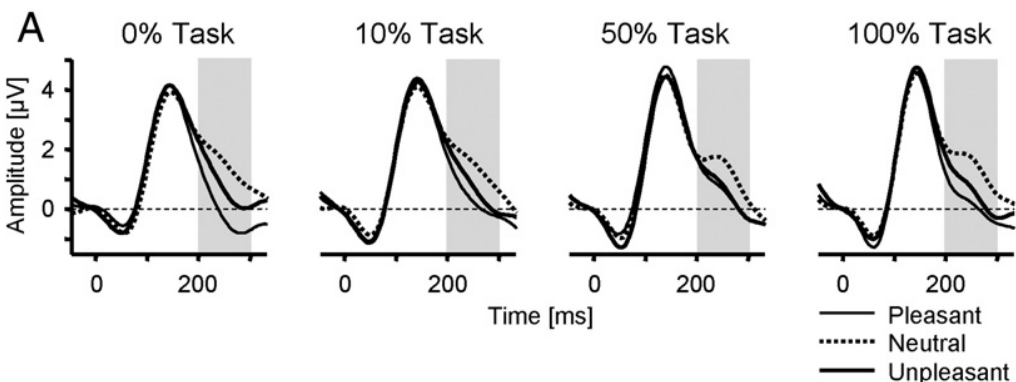

B

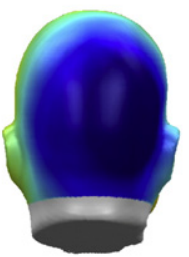

Difference: Pleasant - Neutral
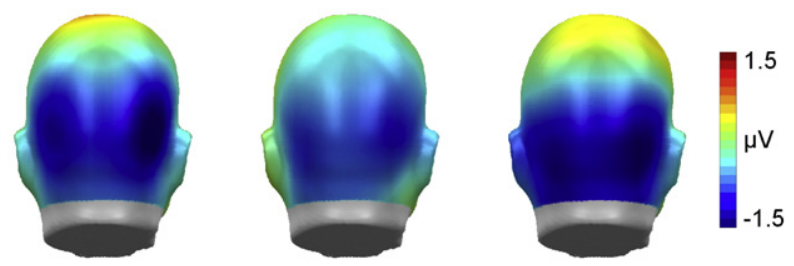

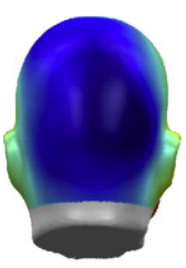

$0 \%$ Task
Difference: Unpleasant - Neutral

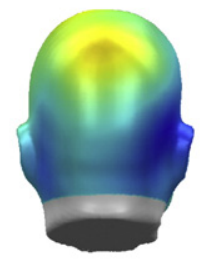

$10 \%$ Task

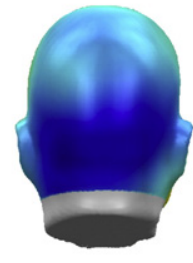

$50 \%$ Task

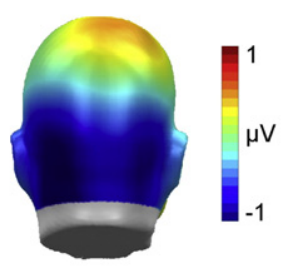

$100 \%$ Task

Fig. 2 - Selective emotion processing as a function of task demand. (A) Grand-averaged ERP waveforms elicited by pleasant, neutral, and unpleasant pictures in the four experimental conditions for a right occipital sensor. The grey-shaded area refers to the analyzed EPN time interval from 200-300 ms. (B) Topographical difference maps for pleasant-neutral and unpleasant-neutral in the four conditions projected on the back view of a model head (mean from 200-300 ms). 
2.3. The interaction of emotion and attention: High-arousing emotional stimulus materials

Previous research provides strong evidence that high arousing emotional materials strongly engage the various emotional response channels measured by fMRI, ERPs, and peripheral physiological measures (Bradley et al., 2001; Sabatinelli et al., 2005). Thus, analyzing high arousing pleasant and unpleasant stimulus materials provides a more powerful test for the inter ference of emotion by competing task demands. In this regard, Schupp and colleagues (Schupp et al., 2007b) observed that the EPN differentiation for high arousing pleasant and unpleasant compared to neutral images was greatly attenuated when participants performed concurrent high demanding visual discrimination tasks. In the present study, the separate ana lysis of high arousing materials revealed markedly different results (see Fig. 3). The main effect of Valence, $F(2,14)$ 17.0, $p<.0001$, was not qualified by an interaction of Valence by Task, $F(6,10)$ 1.0, ns. Separate ANOVAs confirmed highly significant main effects of Valence in all task conditions, Valence $F s(2,14) \quad 9.5,8.8,15.8$, and $11.0, p s<.01$ for the $0 \%, 10 \%$, $50 \%$, and $100 \%$ task condition, respectively. Follow up tests indicated increased EPN amplitudes to pleasant compared to neutral pictures across all conditions, ts(1,15) 4.1, 4.2, 5.8, and $4.8, p s<.001$. Unpleasant compared to neutral pictures were

High Arousing Emotional and Neutral by Task Demand
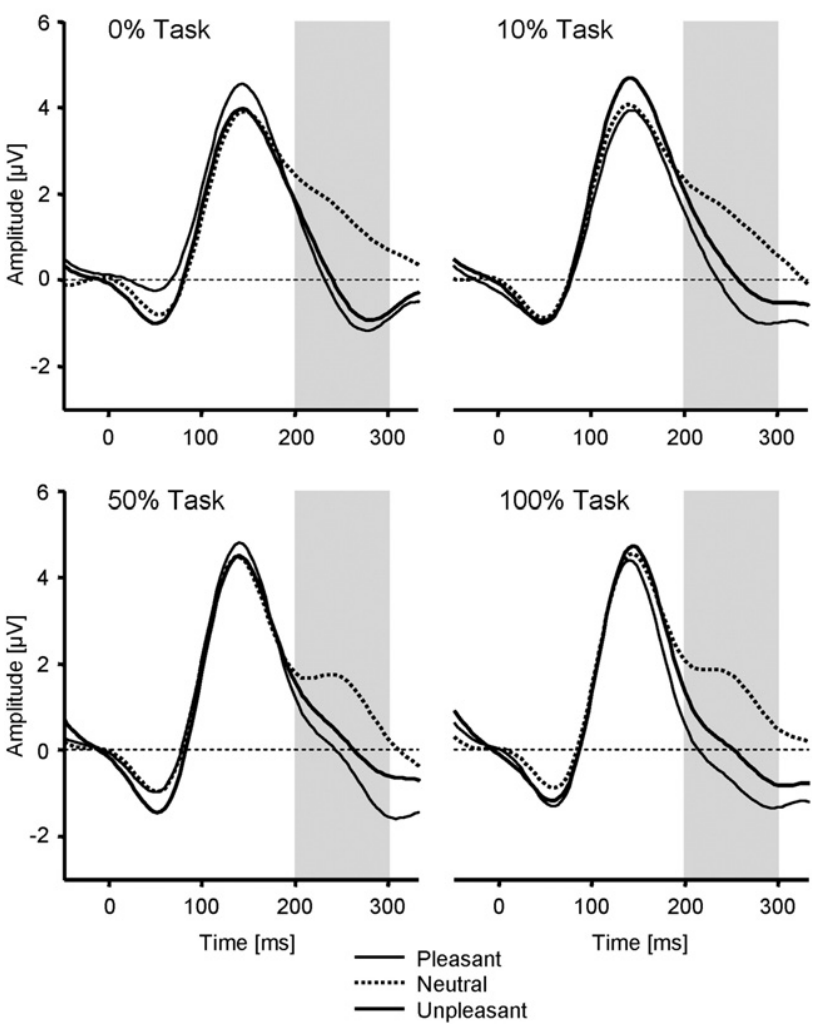

Fig. 3 - Grand-averaged ERP waveforms elicited by high-arousing pleasant, high-arousing unpleasant, and neutral pictures in the four experimental conditions for a right occipital sensor. The grey-shaded area refers to the analyzed EPN time interval from 200-300 ms. associated with increased EPN amplitudes for all task condi tions, ts $(1,15) \quad 4.4,2.2,2.7$, and 3.6, $p s<.05$. Furthermore, larger EPN amplitudes to high arousing pleasant than high arousing unpleasant pictures was observed in the $10 \%$ task condition, $t(1,15) \quad 2.9, p<.01$, and approached significance in the $50 \%$ condition, $t(1,15) \quad 2.0, p$.06.

Furthermore, difference scores of the EPN between high arousing emotional and neutral pictures were calculated for experimental conditions and submitted to repeated measures ANOVA including Task and Emotion Difference. As shown in Fig. 3, emotional differentiation of the EPN was similarly pronounced in all conditions, Task $F(3,13) 1.5$, ns.

\section{Discussion}

Previous studies showed that the processing of emotional compared to neutral pictures is associated with a relative negative difference potential over temporo occipital regions (Junghöfer et al., 2001; Schupp et al., 2006). As expected, these results were fully replicated in the passive viewing condition: Pleasant and unpleasant pictures prompted greater occipital negativity than neutral pictures in a $150300 \mathrm{~ms}$ time window. More interestingly, the present study observed enhanced EPN amplitudes to pleasant and unpleasant pictures in the $50 \%$ and $100 \%$ task conditions, in which participants performed a demanding auditory selective attention task. In addition, selective processing of high arousing emotional picture con tents was similarly expressed across auditory task conditions as in passive picture viewing. Thus, these results revealed little evidence for the attenuation of the EPN emotion modulation when a primary attention task taxed auditory processing resources.

The primary aim of the present study was to precise the locus of interference in emotion processing. The results are consistent with multiple resource theory in which perceptual modality is conceptualized as having separate pools of resources (Wickens, 1980). Furthermore, considered from the perspective of the load theory of attention (Lavie, 2005), the present study provides relevant information regarding the issue whether attention capacity is modality specific or shared between the modalities. Inconsistent with the notion of a supramodal locus of interference, selective emotion proces sing was observed across auditory task conditions. In addition, these findings provide indirect support for the notion that intramodal competition for processing is the primary cause for interference in emotion processing as indexed by the EPN. However, the difficulties in interpreting null findings need to be acknowledged. It has been suggested that in order to observe interference at the perceptual level, available proces sing resources need to be exhausted (Lavie, 2005; Pessoa et al., 2002a). Accordingly, an alternative interpretation of the present findings assumes that task demand was not sufficient to produce interference while more demanding auditory tasks might reveal emotion interference effects. Considering the good behavioral performance, the auditory detection task was not fully exhausting processing resources. However, difficulty ratings indicated that task demands pronouncedly varied across experimental conditions. Furthermore, replicating the classic target P3b effect, the 50 and 100\% task conditions 
revealed enhanced P3 amplitudes to target compared to non target tone stimuli. Overall, while the goal of varying task demand was achieved, the present results are silent with re gard to the issue whether higher levels of auditory task demand interferes with visual emotion processing. Such a pattern of finding emerged in the study of primary visual tasks, in which a moderate task demand did not prevent selective emotion processing while a high task demand abolished emotion discrimination (Vuilleumier et al., 2001; Pessoa et al., 2002b). However, the issue whether selective emotion proces sing is fully immune against auditory task load appears most relevant in the context of strong notions of automaticity. From the perspective of a weakly automatic phenomenon, it ap pears informative to consider the pronounced asymmetry of task modality (visual vs. auditory) in interfering selective emotion processing.

A previous study revealed that a visual discrimination task substantially diminished selective emotion processing (Schupp et al., 2007b). Furthermore, profound interference effects were observed for high arousing stimulus materials, which are particularly efficient in drawing attentional resources (Bradley, 2000). Specifically, the emotional EPN difference was reduced by approximately $50 \%$ during high demand task conditions. In contrast, increasing task demands (50\% and $100 \%$ conditions) in the auditory domain did neither abolish selective emotion processing nor substantially affect the processing of high arousing emotional stimuli. Changing the modality of the primary attention task led to greatly diffe rent outcomes regarding emotion processing although both studies were matched as much as possible in their experi mental design. The same IAPS stimulus materials were shown and emotional and task related stimuli were presented in the same sequence as in the previous study. Furthermore, both tasks were low level discrimination tasks and the counting instruction assured similar load on working memory pro cesses. The main difference between the two studies was task modality: Rather than presenting task related visual stimuli (overlaid on IAPS pictures), high and low frequency tones were presented in the current study. Accordingly, the collective evidence leads to the conclusion that the automatic detection of emotional stimuli is much more affected by concurrent intramodal compared to crossmodal task demands.

In determining the effects of task demands on crossmodal task irrelevant stimulus processing, several further methodo logical issues have been discussed. To prevent the switching of attention across modalities and to assure a high perceptual demand (Muller Grass et al., 2007), visual and auditory stimuli were presented concurrently at fast rates $(3 \mathrm{~Hz})$ rendering these issues unlikely to account for the present findings. A further issue regards the control condition with the recom mendation to compare across different levels of task demand rather than against passive baseline condition (Lavie, 2005). Accordingly, emotional modulation across task conditions is of primary interest. The analysis of emotional difference scores (pleasant neutral, unpleasant neutral) revealed no difference among the three task conditions, supporting the notion that the emotional modulation of the EPN is not subject to pronounced supramodal interference. A further concern is that demanding auditory tasks may have generally increased the amount of processing resources (Kahneman, 1973). While such a hypothesis may accommodate the present findings, it seems difficult to explain the pronounced differences evoked by visual compared to auditory task demands.

The results indicate that pleasant pictures elicited most pronounced EPN amplitudes, being significantly enlarged when compared with unpleasant images. Such a difference in processing pleasant and unpleasant pictures was observed in several previous studies (e.g., De Cesarei et al., 2006), and, most importantly, a strikingly similar difference in EPN ampli tude between emotional materials was observed in the com panion study (Schupp et al., 2007b). The difference is presumably mediated by pictures of erotic content, which elicited most accentuated EPN amplitudes (Schupp et al., 2007b). It has been suggested that features of erotic pictures are easier to recognize and categorize, prompting more efficient encoding and categorization, particularly at these rapid rates (Bradley et al., 2007). Of most interest in the present context, the difference in processing pleasant and unpleasant pictures was most pronounced during passive viewing, possi bly indicating that participants gave priority to pleasant cues in the absence of further instruction and task demands.

The present study specifies boundary conditions for the interference in selective emotion processing. The finding that attentional load in the auditory domain did not interfere with the selective processing of visual emotional stimuli (as indexed by the EPN) is of interest from an ecological perspective. In daily life, attention is often channeled onto a specific perceptual modality raising the issue to which extent emotional stimuli capture attentional resources when pre sented in a different modality. The results indicate that emotional stimuli occurring outside the focus of the attended stimulus modality may capture attentional resources at an early stage of emotional stimulus processing. It has been suggested that the EPN reveals an early "tagging" mechanism of emotionally significant stimuli calling for processing resources in later stages of processing associated with conscious stimulus recognition and focused attention (Schupp et al., 2007b). In this regard, the EPN emotion modulation may increase the probability of an involuntary switch of attention to emotional stimuli due to their implicit stimulus signifi cance. The present findings suggest that this early tagging mechanism is rather immune to crossmodal attentional demands. Recent studies on the ERP correlates of conscious stimulus recognition observed early posterior negativities (150 $300 \mathrm{~ms}$ ) followed by a widely distributed positivity when contrasting perceived with unperceived stimuli (Del Cul et al., 2007; Sergent et al., 2005). Accordingly, future studies may assess both the EPN and the subsequent late positive potential (LPP) to detail the involuntary attention switch and conscious recognition of emotional stimuli presented in an unattended stimulus modality.

In summary, the present findings provide further informa tion regarding the selective processing of emotional cues under conditions of competition. Concurrent processing de mands in the auditory modality did not interfere with selective emotion processing in visual associative brain regions as indexed by the EPN. These findings are consistent with the notion that competition for modality specific perceptual resources may underlie the interference of emotion processing by competing task demands. 


\section{Experimental procedure}

\subsection{Participants}

Sixteen (8 females) right handed introductory psychology stu dents from the University of Greifswald between the ages of 19 and 29 years (M 22.6) participated in the study. The partici pants provided written informed consent for the protocol approved by the Review Board of the University of Greifswald.

\subsection{Experimental design}

Stimulus materials included all 702 pictures of the 1999 International Affective Picture Series (IAPS; Lang et al., 1999). Pleasant, neutral, and unpleasant picture categories differed significantly from each other in their normative valence ratings ( $M$ 6.8, 5.4, and 2.8 for pleasant, neutral, and un pleasant contents on a 19 scale). Mean arousal levels for both emotional categories were significantly higher than for neutral contents ( $M$ 5.2, 3.6, and 5.7 for pleasant, neutral, and unpleasant contents, respectively). Additional analyses explored the processing of pleasant and unpleasant materials high in emotional arousal. Towards this end, 100 high arousing pleasant images (e.g., scenes of erotica, adventure and sports; Valence M 6.5; Arousal M 5.7) and 100 high arousing unpleasant pictures (e.g., scenes of mutilations, threat, and violence; Valence $M$ 2.5; Arousal $M$ 6.1) were selected (Schupp et al., 2007b).

Three out of four experimental conditions included an auditory target discrimination task. Tone stimuli had a frequency of either 1200 or $600 \mathrm{~Hz}$, lasted for $150 \mathrm{~ms}$, and were presented time locked to picture onset (see Fig. 1). The probability of task relevant tones varied across the three task conditions. The $10 \%$ and $50 \%$ task conditions contained 70 and 351 tone stimuli, respectively, randomly distributed over the stream of 702 pictures. In the $100 \%$ condition, task relevant tones were presented concurrently with each picture. In each of the three task conditions, $20 \%$ of the tone stimuli were targets defined as tones of either high or low frequency. Target frequency was balanced across participants. Participants were asked to count the target stimuli and task performance was intermittently assessed during each task condition. Partici pants could earn 5 Euro for correct performance in the counting tasks. Furthermore, a passive viewing condition ( $0 \%$ task) served to replicate previous findings. The order of the four experimental conditions was balanced across participants. Task difficulty was assessed using a 0100 visual analog scale (ranging from very easy to very difficult) for each of the four conditions.

The pictures were presented in all four experimental conditions as continuous stream without perceivable inter stimulus gaps, with each picture shown for $333 \mathrm{~ms}$. Each experimental condition lasted approximately 4 min separated by breaks of about $5 \mathrm{~min}$ between experimental conditions.

\subsection{Data collection, reduction, and analysis}

Brain and ocular scalp potential fields were measured with a 129 lead geodesic sensor net, ensuring an evenly distributed sensor layout over the head surface with an intersensor distance of about $30 \mathrm{~mm}$. Electrode impedance was kept below $30 \mathrm{k} \Omega$. EEG data were recorded continuously with the vertex sensor as reference electrode. The data were on line bandpass filtered from .01 $100 \mathrm{~Hz}$ and sampled at $250 \mathrm{~Hz}$ using Net station software and EGI amplifiers (Electrical Geodesics, Inc., Eugene, Oregon). A $30 \mathrm{~Hz}$ low pass finite impulse response (FIR) filter was applied off line to the continuous EEG data. Stimulus synchronized epochs lasting from $100 \mathrm{~ms}$ before until $800 \mathrm{~ms}$ after picture onset were extracted. Data editing and artifact rejection were based on a method for statistical control of artifacts (Junghöfer et al., 2000). First, global artifacts (e.g., due to movements) were detected by analyzing the data after conversion to an average reference and these trials were excluded from further calculations. Second, individual chan nel artifacts were detected based on the original vertex refe renced data set. Spherical splines (Junghöfer et al., 1997) weighted on the basis of all remaining sensors served to interpolate artifact contaminated individual channels on a trial by trial base. Finally, signal quality was estimated by calculating the variance of the signal across trials. Data repor ted are baseline corrected and converted to an average refe rence (Junghöfer et al., 2006). Emotional modulation of the EPN and target discrimination task effects were explored in two separate streams of analyses. Accordingly, separate average ERP waveforms were calculated for each experimental condi tion and the three picture categories for each sensor and participant. In addition, for the $50 \%$ and $100 \%$ task conditions, average ERP waveforms were calculated as a function of task relevance.

\subsubsection{Selective emotion processing}

Based on previous studies, analyses focused on the assess ment of the Early Posterior Negativity. This emotional ERP modulation appears as a relative negativity compared to neutral materials across different stimulus materials (e.g., IAPS pictures, emotional faces, words and hand gestures) and experimental protocols associated with distinct ERP topographies (Schupp et al., 2006). To capture the EPN, the mean activity over a time interval from 200300 ms was calculated in left and right temporo occipital sensor clusters (EGI sensor numbers; Left: 58, 59, 60, 61, 63, 64, 65, 66, 67, 69, 70, 71, 72, 74, 75; Right: 77, 78, 79, 83, 84, 85, 86, 89, 90, 91, 92, $95,96,97,100)$. The emotional modulation appears reversed in polarity over anterior sites. Exploring anterior sensor sites mirrored the effects observed for the occipital negativity and are not reported here for brevity. To test for statistical significance, a repeated measures ANOVA was calculated including factors of Task $(0,10,50$, and $100 \%$ task conditions), Valence (pleasant, neutral, and unpleasant), and Laterality (left and right).

\subsubsection{Explicit attention task}

A hallmark finding in ERP studies of selective attention is that target compared to non target processing is reflected by in creased P3b amplitudes, i.e., a positive potential shift over centro parietal sensor regions around 300700 ms poststimu lus (Johnson, 1988). The $50 \%$ and $100 \%$ task conditions pro vided a sufficient number of target trials for the reliable assessment of the P3 component. The P3b amplitude was 
scored as mean activity over a time interval from 300400 ms in centro parietal clusters comprising the following sensors of the EGI net: 7, 31, 32, 38, 43, 53, 54, 61 (left hemisphere), and 79, 80, 81, 87, 88, 94, 106, 107 (right hemisphere). P3 scores were submitted in separate analyses for the 50\% and 100\% conditions to repeated measures ANOVA including factors of Target (target vs. non target) and Laterality (left and right).

As in other researches (e.g. Vogel et al., 1998; Sergent et al., 2005), the P3b wave to target pictures was obtained in the presence of the upcoming stimuli. Due to the randomized presentation of IAPS picture categories (pleasant, neutral, and unpleasant), demands of picture processing associated with the subsequent stimuli were kept constant across target and non target conditions. Thus, the target $\mathrm{P} 3 \mathrm{~b}$ wave appears to be related to the attention task. In addition, since the $\mathrm{P} 3 \mathrm{~b}$ component to the target tones occurred at a time at which the following picture has already been displayed, these trials were excluded from the main analyses exploring emotional modulation. Thus, contamination of the ERP waveforms to the IAPS pictures by P3b waves to the preceding target stimuli was circumvented.

\section{Acknowledgment}

This research was supported in part by grants from the German Research Society Institute (Schu1074/7 4 and Schu1074/10 1) to the first author.

\section{R E F E R E N C E S}

Anderson, A.K., Christoff, K., Panitz, D., De Rosa, E., Gabrieli, J.D., 2003. Neural correlates of the automatic processing of threat facial signals. J. Neurosci. 23 (13), 56275633.

Berman, R.A., Colby, C.L., 2002. Auditory and visual attention modulate motion processing in area MTC. Brain. Res. Cogn. Brain. Res. 14, 6474

Bradley, M.M., 2000. Emotion and motivation. In: Cacioppo, J.T., Tassinary, L.G., Berntson, G.G. (Eds.), Handbook of psychophysiology. Cambridge University Press, NY, pp. 602642 .

Bradley, M.M., Codispoti, M., Cuthbert, B.N., Lang, P.J., 2001. Emotion and motivation I: defensive and appetitive reactions in picture processing. Emotion 1 (3), 276298.

Bradley, M.M., Hamby, S., Low, A., Lang, P.J., 2007. Brain potentials in perception: picture complexity and emotional arousal. Psychophysiology 44 (3), 364373.

Cuthbert, B.N., Schupp, H.T., Bradley, M., McManis, M., Lang, P.J., 1998. Probing affective pictures: attended startle and tone probes. Psychophysiology 35 (3), 344347.

Del Cul, A., Baillet, S., Dehaene, S., 2007. Brain dynamics underlying the nonlinear threshold for access to consciousness. PLoS. Biol. 5, e260.

De Cesarei, A., Codispoti, M., 2006. When does size not matter? Effects of stimulus size on affective modulation. Psychophysiology 43 (2), 207215.

Doallo, S., Holguín, S.R., Cadaveira, F., 2006. Attentional load affects automatic emotional processing: evidence from event related potentials. Neuroreport 17, 17971801.

Flaisch, T., Schupp, H.T., Renner, B., Junghöfer, M., submitted for publication. Neural systems of visual attention responding to emotional gestures.
Flaisch, T., Junghöfer, M., Bradley, M.M., Schupp, H.T., Lang, P.J., 2008. Rapid picture processing: Affective primes and targets. Psychophysiology 45, 110.

Hackley, S.A., 1993. An evaluation of the automaticity of sensory processing using event related potentials and brain stem reflexes. Psychophysiology 30, 415428.

Holmes, A., Vuilleumier, P., Eimer, M., 2003. The processing of emotional facial expression is gated by spatial attention: evidence from event related brain potentials. Cogn. Brain. Res. 16 (2), 174184.

Johnson, J.A., Zatorre, R.J., 2005. Attention to simultaneous unrelated auditory and visual events: behavioral and neural correlates. Cereb. Cortex 15 (10), 16091620.

Johnson Jr., R., 1988. The amplitude of the P300 component of the event related potential: review and synthesis. In: Ackles, J.R.J.P.K., Coles, M.G.H. (Eds.), Advances in Psychophysiology, Vol. 3. Jai Press, Greenwich, pp. 69137.

Junghöfer, M., Elbert, T., Leiderer, L., Berg, P., Rockstroh, B., 1997. Mapping EEG potentials on the surface of the brain: a strategy for uncovering cortical sources. Brain. Topogr. 9 (4), 203217.

Junghöfer, M., Elbert, T., Tucker, D.M., Rockstroh, B., 2000. Statistical control of artifacts in dense array EEG/MEG studies. Psychophysiology 37 (4), 523532

Junghöfer, M., Bradley, M.M., Elbert, T.R., Lang, P.J., 2001. Fleeting images: a new look at early emotion discrimination. Psychophysiology 38 (2), 175178.

Junghöfer, M., Schupp, H.T., Stark, R., Vaitl, D., 2005. Neuroimaging of emotion: empirical effects of proportional global signal scaling in fMRI data analysis. Neuroimage 25 (2), 520526.

Junghöfer, M., Peyk, P., Flaisch, T., Schupp, H.T., 2006. Neuroimaging methods in affective neuroscience: selected methodological issues. Prog. Brain. Res. 156, 123143.

Kahneman, D., 1973. Attention and Effort. Prentice Hall, Engiewood Cliffs, NJ.

Kahneman, D., Treisman, A.M., 1984. Changing views of attention and automaticity. In: Parasuraman, R., Davies, R. (Eds.), Varieties of attention, NJ, pp. 2961.

Keil, A., Bradley, M.M., Junghöfer, M., Russmann, T., Lowenthal, W., Lang, P.J., 2007. Cross modal attention capture by affective stimuli: evidence from event related potentials. Cogn. Affect. Behav. Neurosci. 7 (1), 1824.

Kissler, J., Herbert, C., Peyk, P., Junghöfer, M., 2007. Buzzwords: early cortical responses to emotional words during reading. Psychol. Sci. 18 (6), 475480.

Kramer, A.F., Trejob, L.J., Humphrey, D., 1995. Assessment of mental workload with task irrelevant auditory probes. Biol. Psychol. 40 (1 2), 83100.

Lang, P.J., Bradley, M.M., Cuthbert, B.N., 1997. Motivated attention: affect, activation, and action. In: Lang, P.J., Simons, R.F., Balaban, M. (Eds.), Attention and Emotion: Sensory and Motivational Processes. Erlbaum, Mahwah, NJ, pp. 97135.

Lang, P.J., Bradley, M.M., Fitzsimmons, J.R., Cuthbert, B.N., Scott, J.D., Moulder, B., et al., 1998. Emotional arousal and activation of the visual cortex: an fMRI analysis. Psychophysiology 35 (2), 199210.

Lang, P.J., Bradley, M.M., Cuthbert, B.N., 1999. International affective picture system (IAPS): instruction manual and affective ratings. Technical Report A 4. The Center for Research in Psychophysiology, University of Florida, Gainesville, FL.

Lavie, N., 2005. Distracted and confused? Selective attention under load. Trends. Cogn. Sci. 9 (2), 7582.

Mathews, A., MacLeod, C., 1994. Cognitive approaches to emotion and emotional disorders. Annu. Rev. Psychol. 45, 2550.

Mogg, K., Bradley, B.P., 1999. Some methodological issues in assessing attentional biases for threatening faces in anxiety: a replication study using a modified version of the probe detection task. Behav. Res. Ther. 37 (6), 595604.

Muller Gass, A., Macdonald, M., Schröger, E., Sculthorpe, L., Campbell, K., 2007. Evidence for the auditory P3a reflecting an 
automatic process: elicitation during highly focused continuous visual attention. Brain. Res. 1170, 7178.

Öhman, A., Flykt, A., Lundqvist, D., 2000. Unconscious emotion: evolutionary perspectives, psychophysiological data and neuropsychological mechanisms. In: Lane, R.D., Nadel, L. (Eds.), Cognitive Neuroscience of Emotion. Oxford University Press, NY, pp. 296327.

Pessoa, L., Kastner, S., Ungerleider, L.G., 2002a. Attentional control of the processing of neutral and emotional stimuli. Cogn. Brain. Res. 15 (1), 3145.

Pessoa, L., McKenna, M., Gutierrez, E., Ungerleider, L.G., 2002b. Neural processing of emotional faces requires attention. Proc. Natl. Acad. Sci. U. S. A. 99 (17), 1145811463.

Rees, G., Frith, C., Lavie, N., 2001. Perception of irrelevant visual motion during performance of an auditory attention task. Neuropsychologia 39, 937949.

Sabatinelli, D., Flaisch, T., Bradley, M.M., Fitzsimmons, J.R., Lang, P.J., 2004. Affective picture perception: gender differences in visual cortex? Neuroreport 15 (7), 11091112.

Sabatinelli, D., Bradley, M.M., Fitzsimmons, J.R., Lang, P.J., 2005. Parallel amygdala and inferotemporal activation reflect emotional intensity and fear relevance. Neuroimage 24 (4), 12651270

Schupp, H.T., Junghöfer, M., Weike, A.I., Hamm, A.O., 2003. Attention and emotion: an ERP analysis of facilitated emotional stimulus processing. Neuroreport 14 (8), 11071110.

Schupp, H.T., Cuthbert, B.N., Bradley, M.M., Hillman, C.H., Hamm, A.O., Lang, P.J., 2004a. Brain processes in emotional perception: motivated attention. Cognition and Emotion 18 (5), 593611.

Schupp, H.T., Öhman, A., Junghöfer, M., Weike, A.I., Stockburger, J., Hamm, A.O., 2004b. The facilitated processing of threatening faces: an ERP analysis. Emotion 4 (2), 189200.
Schupp, H.T., Flaisch, T., Stockburger, J., Junghöfer, M., 2006. Emotion and attention: event related brain potential studies. Prog. Brain. Res. 156, 3151.

Schupp, H.T., Stockburger, J., Bublatzky, F., Junghöfer, M., Weike, A.I., Hamm, A.O., 2007a. Explicit attention interferes with selective emotion processing in human extrastriate cortex. BMC. Neurosci. 8 (1).

Schupp, H.T., Stockburger, J., Codispoti, M., Junghöfer, M., Weike, A.I., Hamm, A.O., 2007b. Selective visual attention to emotion. J. Neurosci. 27 (5), 10821089.

Sergent, C., Baillet, S., Dehaene, S., 2005. Timing of the brain events underlying access to consciousness during the attentional blink. Nat. Neurosci. 8 (10), 13911400.

Surakka, V., Tenhunen Eskelinen, M., Hietanen, J.K., Sams, M., 1998. Modulation of human auditory information processing by emotional visual stimuli. Cogn. Brain. Res. 7, 159163.

Vogel, E.K., Luck, S.J., Shapiro, K.L., 1998. Electrophysiological evidence for a postperceptual locus of suppression during the attentional blink. J. Exp. Psychol. Hum. Percept. Perform. 24 (6), 16561674.

Vuilleumier, P., Armony, J.L., Driver, J., Dolan, R.J., 2001. Effects of attention and emotion on face processing in the human brain: an event related fMRI study. Neuron 30 (3), 829841.

Wickens, C.D., 1980. The structure of attentional resources In: Nickerson, R. (Ed.), Attention and performance VIII., Hillsdale NJ, pp. 239257.

Woldorff, M.G., Hackley, S.A., Hillyard, S.A., 1991. The effects of channel selective attention on the mismatch negativity wave elicited by deviant tones. Psychophysiology. 28,3042 . 